\title{
Commentary
}

\section{Venous ulcer: Current concepts}

\section{James Roy Kanjoor}

Canadian Medical Center, Kuwait

Address for correspondence: Dr. James Roy Kanjoor, Canadian Medical Center, Kuwait. E-mail: roykanjoor@yahoo.com

he high prevalence of the venous ulceration, its tendency for recurrence, and above all, the ineffectiveness of treatments make them the subject of many a research. They affect both the quality of life of the sufferers as well as their productivity at work places.

\begin{tabular}{|l|l|}
\hline \multicolumn{3}{|c|}{ Access this article online } \\
\hline Quick Response Code: & Website: \\
\hline & www.ijps.org \\
\cline { 2 - 2 } & DOI: \\
\hline
\end{tabular}

Proper understanding of the pathophysiology and the clinical behaviour of chronic venous insufficiency, which leads to venous ulceration, is essential for a rational approach towards these patients.

Venous ulcers are generally irregular, shallow, and located over bony prominences. Granulation tissue and fibrin are typically present in the ulcer base. Associated findings include lower extremity varicosities, oedema ankle flare, venous dermatitis, and lipodermatosclerosis. Venous ulcers are usually recurrent, and an open ulcer can persist for weeks to many years. Severe complications include cellulitis, osteomyelitis, and malignant change 


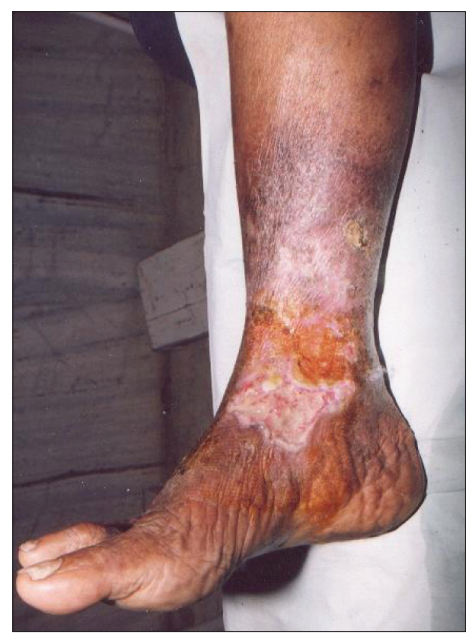

Figure 1: Chronic venous ulcer with malignant transformation - Marjolin's ulcer. Note the zone of lipodermatosclerosis proximal to the ulcer and ankle flare proximal to that

[Figure 1]. Poor prognostic factors include large ulcer size and prolonged duration. ${ }^{[1]}$

The contribution of venous system towards healing of a leg ulcer is vital. Calf muscle pump dysfunction impairs this healing as does valvular incompetence as seen in post thrombotic venous recanalization and in instances of perforator incompetence. The basic pathology is venous hypertension affecting the macrocirculation with its resultant effects on dermal microcirculation..$^{[2]}$

Venous blood flows through perforating veins and follows the pressure gradient from elevated hydrostatic pressures in the superficial veins-great and small saphenous veins to the decreased pressure in the deep veins. Abnormal pressure in either the superficial or deep veins will result in venous hypertension.

Decreased ankle range of motion is associated with poor calf pump function and correlates with the clinical progression to venous disease and ulceration.

Reflux and venous outflow obstruction result in the development of venous hypertension. Venous reflux can be primary valvular reflux or secondary to venous thrombosis and concomitant valvular destruction. The development of venous hypertension initiates a cascade of pathological events leading to lower extremity oedema, pain, itching, dermatitis, skin discolouration (ankle flare), varicosities, and, finally, venous ulceration. A combination of both reflux and obstruction is observed in about $17 \%$ of venous ulcerations. Isolated venous outflow obstruction is associated with venous ulcer formation in $1-6 \%$ of patients.
More than $85 \%$ of patients with chronic venous insufficiency have disease in their superficial veins. This is important, because it has been shown that when venous ulceration is due to superficial and perforator vein incompetence alone, surgical treatment may heal up to $90 \%$ of ulcers.

Johnson et al. ${ }^{[3]}$ reported that after an episode of acute deep vein thrombosis, $12 \%$ of limbs return to normal by duplex criteria and $41 \%$ have anatomical features of post thrombotic syndrome, despite the fact that only $13 \%$ develop skin complications.

Haemodynamic forces associated with chronic venous hypertension cause permeability alterations in skin's microcirculation. Extravasation of intraluminal red blood cells and macromolecules into interstitium represents the chronic injury stimulus leading to endothelial activation, white blood cell chemotaxis and inflammation induced injury. The number of leucocytes in the dermis of patients correlates with the clinical disease severity. There is interplay of many chemical mediators like TGF-B1, matrix metallo proteases (MMPs) and their inhibitors. The end result of these inflammatory events is fibrotic and oedematous skin, and injury to nutrient and exchange capillaries. The slightest trauma or infection in these areas results in an imbalance of tissue remodelling that leads to dermal fibrosis and ulcer formation.

Thus, initial examination should include an arterial pulse and Doppler examination. The next step is the duplex scan with colour flow, which is the mainstay of non invasive evaluation. It provides real-time observation of venous flow in the leg, the effect of muscle contraction, proximal and distal compression and the Valsalva maneuver on each segment of the veins. The veins can also be assessed for patency and reflux.

Air plethysmography provides quantitative data on obstruction, calf muscle pump ejection fraction and reflux.

Venous pressure studies are helpful in assessing the physiological importance of anatomic obstruction, because the collaterals may or may not provide adequate compensation for an obstructed pathway. Plethysmography and pressure data are important when trying to determine the need for surgical bypass or valve replacement. 
Ascending and descending venography are important in candidates who are being considered for deep vein reconstruction.

A common cause for prolonged wound healing is misdiagnosis of an ulcer as a venous ulcer. Lack of improvement of an ulcer with good local wound care over a 4-week period mandates a biopsy to be performed to rule out malignancy. With conservative methods, the mean ulcer healing time is 5.3 months. ${ }^{[4]}$

While treating venous ulcers the twin goals that need to be achieved are ulcer healing and prevention of its recurrence. This is indeed challenging, even to the Plastic Surgeons. ${ }^{[5]}$ The main stay is conservative care by good dressing, elevation and compression therapy. Even when surgically correctable venous pathology is managed, the patient has to carry on with conservative treatment and compression garments lifelong to prevent recurrences.

Pentoxifylline, micronised purified flavanoid fractionDaflon $500 \mathrm{mg}$ and prostaglandin E1 analogue are the drugs used due to their action on leucocyte metabolism. The drugs are most effective when used in conjunction with compression. ${ }^{[2]}$

Many evidence-based studies conclude that compression increases ulcer healing rates compared with no compression. Multi-component systems are more effective than single-component systems. Multicomponent systems containing an elastic bandage appear more effective than those composed mainly of inelastic constituents. ${ }^{[6]}$

It has been shown that more than $85 \%$ of patients with leg ulcer have reflux in superficial veins and therefore are candidates for intervention.

Sclerotherapy, endoscopic or other methods of subfascial ligation of incompetent communicating/perforator veins, vein stripping, endovenous laser obliteration, and primary venous and valvular reconstruction target the primary venous aetiology.

Surgery for the ulcers is indicated when they are large in size, of a prolonged duration and refractory to conservative treatment. ${ }^{[7,8]}$ Hospitalization, wound bed preparation and appropriate skin cover by a skin graft or flap is advised. Limb elevation, dressings using any of the biological dressings, growth factors and VAC have been reported to accelerate healing. Areas of lipodermatosclerosis should be excised and the perforator veins need be ablated before any skin graft or flap transfer.

In conclusion, venous disease is the most common cause of leg ulceration. Early diagnosis and prevention are essential. Managing the primary venous pathology is the crucial step to prevent the recurrence of a venous ulcer, which has healed by the conventional wound care. Proper compressive garments have to be worn lifelong.

\section{REFERENCES}

1. Collins L, Seraj S. Diagnosis and treatment of venous ulcers. Am Fam Physician 2010;81:989-9.

2. Patel NM, Labropoulos N, Pappas PJ. Current management of venous Ulceration. Plast Reconst Surg 2006;117:254-60S.

3. Johnson BF, Manzo RA, Bergelin RO, Strandness DE Jr. Relationship between changes in the deep venous system and the development of the post thrombotic syndrome after an acute episode of lower limb deep vein thrombosis: a one to six year follow up. J Vasc Surg 1995;21:307-12.

4. Nicoloff AD, Moneta GL, Porter JM. Compression treatment of Chronic Venous Insufficiency. In: Glovicki P, Yao JS, editors. Handbook of Venous disorders. $2^{\text {nd }}$ ed. Guidelines of the American Venous Forum. Arnold; 2001.p. 303-8.

5. Alamelu V. Is chronic venous ulcer curable? A sample survey of a plastic surgeon. Indian J Plast Surg; 2011;44:104-9.

6. O'Meara S, Cullum NA, Nelson EA. Compression for venous leg ulcers. Cochrane Database Syst Rev 2009;1:CD000265.

7. Reddy MM, Reddy MD. Role of free tissue transfer in the management of chronic venous ulcer. Indian J Plast Surg 2004;37:28-33.

8. Kumins $\mathrm{NH}$, Weinzweig $\mathrm{N}$, Schler JJ. Free tissue transfer provides durable treatment for large non healing venous ulcers. J Vasc Surg 2000;32:848-54. 